\title{
Every planar graph without cycles of lengths 4 to 12 is acyclically 3 -choosable
}

\author{
Hervé Hocquard and Mickaël Montassier \\ LaBRI, Université Bordeaux I, 33405 Talence Cedex, France
}

June 4, 2009

\begin{abstract}
An acyclic coloring of a graph $G$ is a coloring of its vertices such that : (i) no two adjacent vertices in $G$ receive the same color and (ii) no bicolored cycles exist in $G$. A list assignment of $G$ is a function $L$ that assigns to each vertex $v \in V(G)$ a list $L(v)$ of available colors. Let $G$ be a graph and $L$ be a list assignment of $G$. The graph $G$ is acyclically $L$-list colorable if there exists an acyclic coloring $\phi$ of $G$ such that $\phi(v) \in L(v)$ for all $v \in V(G)$. If $G$ is acyclically $L$-list colorable for any list assignment $L$ with $|L(v)| \geq k$ for all $v \in V(G)$, then $G$ is acyclically $k$-choosable. In this paper, we prove that every planar graph without cycles of lengths 4 to 12 is acyclically 3-choosable.
\end{abstract}

\section{Introduction}

A proper coloring of a graph is an assignment of colors to the vertices of the graph such that two adjacent vertices do not use the same color. A $k$-coloring of $G$ is a proper coloring of $G$ using $k$ colors ; a graph admitting a $k$-coloring is said to be $k$-colorable. An acyclic coloring of a graph $G$ is a proper coloring of $G$ such that $G$ contains no bicolored cycles ; in other words, the graph induced by every two color classes is a forest. A list assignment of $G$ is a function $L$ that assigns to each vertex $v \in V(G)$ a list $L(v)$ of available colors. Let $G$ be a graph and $L$ be a list assignment of $G$. The graph $G$ is acyclically L-list colorable if there is an acyclic coloring $\phi$ of $G$ such that $\phi(v) \in L(v)$ for all $v \in V(G)$. If $G$ is acyclically $L$-list colorable for any list assignment $L$ with $|L(v)| \geq k$ for all $v \in V(G)$, then $G$ is acyclically $k$-choosable. The acyclic choice number of $G, \chi_{a}^{l}(G)$, is the smallest integer $k$ such that $G$ is acyclically $k$-choosable. Borodin $e t$ al. [1] first investigated the acyclic choosability of planar graphs proving that:

Theorem 1 [1] Every planar graph is acyclically 7-choosable.

and put forward to the following challenging conjecture:

Conjecture 1 [1] Every planar graph is acyclically 5-choosable.

This conjecture if true strengthens Borodin's Theorem [3] on the acyclic 5-colorability of planar graphs and Thomassen's Theorem [11] on the 5-choosability of planar graphs.

In 1976, Steinberg conjectured that every planar graph without cycles of lengths 4 and 5 is 3 colorable (see Problem 2.9 [7]). This problem remains open. In 1990, Erdôs suggested the following relaxation of Steinberg's Conjecture: what is the smallest integer $i$ such that every planar graph without cycles of lengths 4 to $i$ is 3 -colorable? The best known result is $i=7$ [2]. This question is also studied in the choosability case: what is the smallest integer $i$ such that every planar graph without cycles of lengths 4 to $i$ is 3-choosable? In [12], Voigt proved that Steinberg's Conjecture can not be extended to list coloring; hence, $i \geq 6$. Nevertheless, in 1996, Borodin [4] proved that every planar graph without cycles of lengths 4 to 9 is 3 -colorable; in fact, 3 -choosable. So, $i \leq 9$.

In this paper, we study the question of Erdôs in the acyclic choosability case: 
Problem 1 What is the smallest integer $i$ such that every planar graph without cycles of lengths 4 to $i$ is acyclically 3 -choosable?

Note that it is proved that every planar graph without cycles of lengths 4 to 6 is acyclically 4 choosable [10]. Also, the relationship between the maximum average degree of $G$ (or the girth of $G$ ) and its acyclic choice number was studied (see for example $[9,8,5]$ ).

Our main result is the following:

Theorem 2 Every planar graph without cycles of lengths 4 to 12 is acyclically 3-choosable.

Hence, in Problem $1,6 \leq i \leq 12$.

Section 2 is dedicated to the proof of Theorem 2. Follow some notations we will use:

Notations Let $G$ be a planar graph. We use $V(G), E(G)$ and $F(G)$ to denote the set of vertices, edges and faces of $G$ respectively. Let $d(v)$ denote the degree of a vertex $v$ in $G$ and $r(f)$ the length of a face $f$ in $G$. A vertex of degree $k$ (resp. at least $k$, at most $k$ ) is called a $k$-vertex (resp. $\geq k$ vertex, $\leq k$-vertex). We use the same notations for faces : a $k$-face (resp. $\geq k$-face, $\leq k$-face) is a face of length $k$ (resp. at least $k$, at most $k$ ). A $k$-face having the boundary vertices $x_{1}, x_{2}, \ldots, x_{k}$ in the cyclic order is denoted by $\left[x_{1} x_{2} \ldots x_{k}\right]$. For a vertex $v \in V(G)$, let $n_{i}(v)$ denote the number of $i$-vertices adjacent to $v$ for $i \geq 1$, and $m_{3}(v)$ the number of 3-faces incident to $v$. A 3-vertex is called $3^{*}$-vertex if it is incident to a 3 -face and adjacent to a 2-vertex (for example in Figure 1, the vertex $t$ is a $3^{*}$-vertex). A 3 -face $[r s t]$ with $d(r)=d(s)=d(t)=3$ and with a $3^{*}$-vertex on its boundary is called a $3^{*}$-face. Two 3-faces $[$ rst $]$ and $[u v w]$ are called linked if there exists an edge $t v$ which connects these two 3-faces such that $d(t)=d(v)=3$ (see Figure 2). A vertex $v$ is linked to a 3-face $[r s t]$ if there exists an edge between $v$ and one vertex of the boundary of $[r s t]$, say $t$, such that $d(t)=3$ (for example in Figure 1, the vertex $v$ is linked to the 3-face $[r s t]$ ). Let $n^{*}(v)$ be the number of $3 *$-face linked to $v$.

$S$

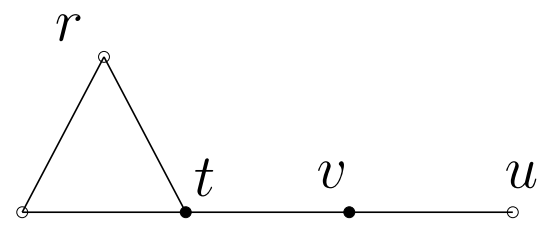

Figure 1: The vertex $t$ is a $3^{*}$-vertex and the vertex $v$ is linked to the 3 -face $[r s t]$

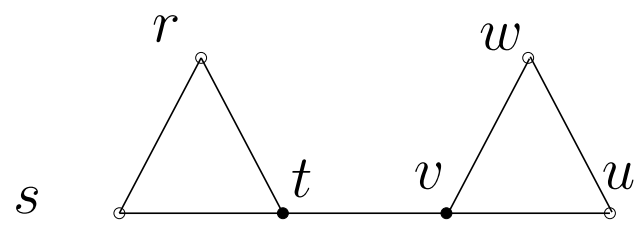

Figure 2: The two 3-faces [rst] and [uvw] are linked

\section{Proof of Theorem 2}

To prove Theorem 2 we proceed by contradiction. Suppose that $H$ is a counterexample with the minimum order to Theorem 2 which is embedded in the plane. Let $L$ be a list assignment with $|L(v)|=3$ for all $v \in V(H)$ such that there does not exist an acyclic coloring $c$ of $H$ with for all $v \in V(H), c(v) \in L(v)$.

Without loss of generality we can suppose that $H$ is connected. We will first investigate the structural properties of $H$ (Section 2.1), then using Euler's formula and the discharging technique we will derive a contradiction (Section 2.2). 


\subsection{Structural properties of $H$}

Lemma 1 The minimal counterexample $H$ to Theorem 2 has the following properties:

(C1) H contains no 1-vertices.

(C2) A 3-face has no 2-vertex on its boundary.

(C3) A 2-vertex is not adjacent to a 2-vertex.

(C4) A 3-face has at most one $3^{*}$-vertex on its boundary.

(C5) A 3-face $[r s t]$ with $d(r)=d(s)=d(t)=3$ is linked to at most one $3^{*}$-face.

(C6) Two $3^{*}$-faces cannot be linked.

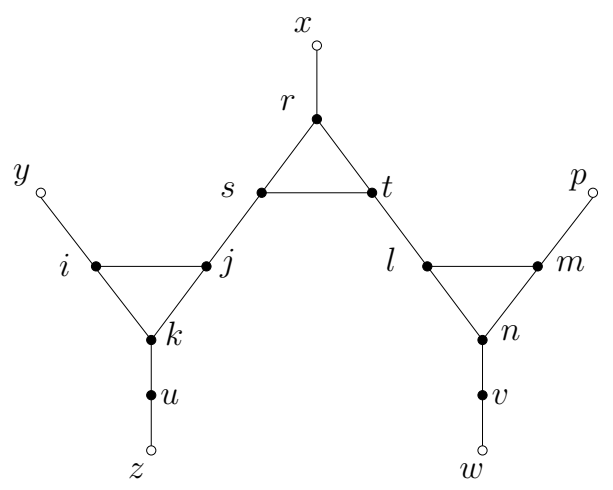

Figure 3: $[r s t]$ is linked to two $3^{*}$-faces $[i j k]$ and $[l m n]$

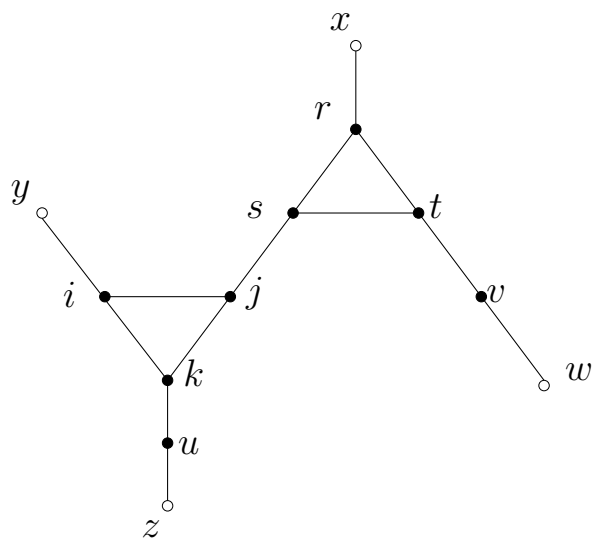

Figure 4: The two $3^{*}$-faces $[r s t]$ and $[i j k]$ are linked

\section{Proof}

(C1) Suppose $H$ contains a 1-vertex $u$ adjacent to a vertex $v$. By minimality of $H$, the graph $H^{\prime}=H \backslash\{u\}$ is acyclically 3 -choosable. Consequently, there exists an acyclic $L$-coloring $c$ of $H^{\prime}$. To extend this coloring to $H$ we just color $u$ with $c(u) \in L(u) \backslash\{c(v)\}$. The obtained coloring is acyclic, a contradiction. 
(C2) Suppose $H$ contains a 2-vertex $u$ incident to a 3-face [uvw]. By minimality of $H$, the graph $H^{\prime}=H \backslash\{u\}$ is acyclically 3 -choosable. Consequently, there exists an acyclic $L$-coloring $c$ of $H^{\prime}$. We show that we can extend this coloring to $H$ by coloring $u$ with $c(u) \in L(u) \backslash\{c(v), c(w)\}$.

(C3) Suppose $H$ contains a 2-vertex $u$ adjacent to a 2-vertex $v$. Let $t$ and $w$ be the other neighbors of $u$ and $v$ respectively. By minimality of $H$, the graph $H^{\prime}=H \backslash\{u\}$ is acyclically 3 -choosable. Consequently, there exists an acyclic $L$-coloring $c$ of $H^{\prime}$. We show that we can extend this coloring to $H$. Assume first that $c(t) \neq c(v)$. Then we just color $u$ with $c(u) \in L(u) \backslash\{c(t), c(v)\}$. Now, if $c(t)=c(v)$, we color $u$ with $c(u) \in L(u) \backslash\{c(v), c(w)\}$. In the two cases, the obtained coloring is acyclic, a contradiction.

(C4) Suppose $H$ contains a 3 -face $[r s t]$ with two $3^{*}$-vertices $s$ and $t$. Suppose that $t$ (resp. $s$ ) is adjacent to a 2-vertex $v$ (resp. $x$ ) with $v \neq r, s$ by (C2) (resp. $x \neq r, t$ ). Let $u$ (resp. $y$ ) be the other neighbor of $v$ (resp. $x$ ) with $u \neq r, s$ (resp. $y \neq r, t$ ). By the minimality of $H, H^{\prime}=$ $H \backslash\{v\}$ is acyclically 3 -choosable. Consequently, there exists an acyclic $L$-coloring $c$ of $H^{\prime}$. We show now that we can extend $c$ to $H$. If $c(u) \neq c(t)$, we color then $v$ with a color different from $c(u)$ and $c(t)$ and the coloring obtained is acyclic. Otherwise, $c(u)=c(t)$. If we cannot color $v$, this implies without loss of generality $L(v)=\{1,2,3\}, c(u)=c(t)=c(x)=1$, $c(r)=2$ and $c(s)=c(y)=3$. Observe that necessarily $L(t)=\{1,2,3\}$ (otherwise we can recolor $t$ with $\alpha \in L(t) \backslash\{1,2,3\}$ and color $v$ properly i.e $v$ receives a color distinct of those of these neighbors). For a same reason $L(s)=\{1,2,3\}$ and $L(x)=\{1,2,3\}$. Now, we recolor $t$ with the color $3, s$ with the color 1 and $x$ with the color 2, then we can color $v$ with the color 2. It is easy to see that the coloring obtained is acyclic.

(C5) Suppose $H$ contains a 3-face $[r s t]$ incident to three 3-vertices such that two of them are linked to two $3^{*}$-faces $[i j k]$ and $[l m n]$. Suppose $[i j k]$ and $[l m n]$ are linked to $[r s t]$ respectively by the edges $s j$ and $t l$. Call $y$ the third neighbor of $i, x$ the third neighbor of $r$, and $p$ the third neighbor of $m$. Suppose that the 2-vertex $u$ (resp. $v$ ) is adjacent to $k$ and $z$ (resp. $n$ and $w$ ). For example, $H$ contains the graph depicted by Figure 3. By the minimality of $H$, $H^{\prime}=H \backslash\{v\}$ is acyclically 3 -choosable. Consequently, there exists an acyclic $L$-coloring $c$ of $H^{\prime}$. We show now that we can extend $c$ to $H$. If $c(w) \neq c(n)$, we color then $v$ with a color different from $c(w)$ and $c(n)$ and the coloring obtained is acyclic. Otherwise, $c(w)=c(n)$. If we cannot color $v$, this implies without loss of generality $L(v)=\{1,2,3\}=L(l)=L(m)$, $c(w)=c(n)=c(t)=c(p)=1$, and by permuting the colors of $l$ and $m$, we are sure that $L(r)=\{1,2,3\}=L(s)$ and $c(x)=c(j)=1$, then by permuting the colors of $r$ and $s$, we are sure that $L(i)=\{1,2,3\}=L(k), c(y)=c(u)=1$, and $c(z) \in\{2,3\}$. Let $\alpha=\{2,3\} \backslash\{c(z)\}$. We recolor $k, s, l, v$ with $\alpha$ and $m, r, i$ with $c(z)$. The coloring obtained is acyclic.

(C6) Suppose $H$ contains a 3-face $[r s t]$ incident to three 3-vertices such that one vertex is linked to a $3^{*}$-face, say $s$ is linked by the edge $s j$ to the $3^{*}$-face $[i j k]$ and one vertex is a $3^{*}$-vertex, say $t$. Call $y$ the third neighbor of $i, x$ the third neighbor of $r$. Suppose that the 2-vertex $u$ (resp. $v$ ) is adjacent to $k$ and $z$ (resp. $t$ and $w$ ). For example, $H$ contains the graph depicted by Figure 4. By the minimality of $H, H^{\prime}=H \backslash\{v\}$ is acyclically 3-choosable. Consequently, there exists an acyclic $L$-coloring $c$ of $H^{\prime}$. We show now that we can extend $c$ to $H$. If $c(w) \neq c(t)$, we color then $v$ with a color different from $c(w)$ and $c(t)$ and the coloring obtained is acyclic. Otherwise, $c(w)=c(t)$. If we cannot color $v$, this implies without loss of generality $L(v)=\{1,2,3\}=L(r)=L(s), c(w)=c(t)=c(x)=c(j)=1$, and by permuting the colors of $r$ and $s$, we are sure that $L(i)=\{1,2,3\}=L(k), c(y)=c(u)=1$, and $c(z) \in\{2,3\}$. Let $\alpha=\{2,3\} \backslash\{c(z)\}$. We recolor $k, s, v$ with $\alpha$ and $r, i$ with $c(z)$. The coloring obtained is acyclic.

Lemma 2 Let $H$ be a connected plane graph with $n$ vertices, $m$ edges and $r$ faces. Then, we have 
the following:

$$
\sum_{v \in V(H)}(11 d(v)-26)+\sum_{f \in F(H)}(2 r(f)-26)=-52
$$

Proof

Euler's formula $n-m+f=2$ can be rewritten as $(22 m-26 n)+(4 m-26 f))=-52$. The relation $\sum_{v \in V(H)} d(v)=\sum_{f \in F(H)} r(f)=2 m$ completes the proof.

\subsection{Discharging procedure}

Let $H$ be a counterexample to Theorem 2 with the minimum order. Then, $H$ satisfies Lemma 1 .

We define the weight function $\omega: V(H) \cup F(H) \longrightarrow \mathbb{R}$ by $\omega(x)=11 d(x)-26$ if $x \in V(H)$ and $\omega(x)=2 r(x)-26$ if $x \in F(H)$. It follows from Equation (1) that the total sum of weights is equal to -52. In what follows, we will define discharging rules (R1) and (R2) and redistribute weights accordingly. Once the discharging is finished, a new weight function $\omega^{*}$ is produced. However, the total sum of weights is kept fixed when the discharging is achieved. Nevertheless, we will show that $\omega^{*}(x) \geq 0$ for all $x \in V(H) \cup F(H)$. This leads to the following obvious contradiction:

$$
0 \leq \sum_{x \in V(H) \cup F(H)} \omega^{*}(x)=\sum_{x \in V(H) \cup F(H)} \omega(x)=-52<0
$$

and hence demonstrates that no such counterexample can exist.

The discharging rules are defined as follows:

(R1.1) Every $\geq 3$-vertex $v$ gives 2 to each adjacent 2-vertex.

(R1.2) Every $\geq 4$-vertex $v$ gives 9 to each incident 3 -face and 1 to each linked $3{ }^{*}$-face.

(R2.1) Every $3^{*}$-vertex $v$ gives 5 to its incident 3 -face.

(R2.2) Every 3-vertex $v$, different from a $3^{*}$-vertex, which is not linked to a $3^{*}$-face, gives 7 to its incident 3-face (if any).

(R2.3) Every 3 -vertex $v$, different from a $3^{*}$-vertex, linked to a $3^{*}$-face gives 1 to each linked $3^{*}$-face and gives 6 to its incident 3 -face (if any).

In order to complete the proof, it suffices to prove that the new weight $\omega^{*}(x)$ is non-negative for all $x \in V(H) \cup F(H)$.

Let $v \in V(H)$ be a $k$-vertex. Then, $k \geq 2$ by (C1).

- If $k=2$, then $\omega(v)=-4$ and $v$ is adjacent to two $\geq 3$-vertices by (C3). By (R1.1), $\omega^{*}(v)=$ $-4+2 \cdot 2=0$.

- If $k=3$, then $\omega(v)=7$. Since $H$ contains no 4-cycles, $v$ is incident to at most one 3-face. Assume first that $v$ is not incident to a 3-face. Then by (R1.1) and (R2.3), $v$ gives at most 3 times 2. Hence, $\omega^{*}(v) \geq 7-3 \cdot 2 \geq 1$. Assume now that $v$ is incident to a 3 -face. If $v$ is a $3^{*}$-vertex, then $\omega^{*}(v)=7-5-2=0$ by (R1.1) and (R2.1). If $v$ is linked to a $3^{*}$-face then $\omega^{*}(v) \geq 7-6-1=0$ by (R2.3). If $v$ is not adjacent to a 2 -vertex and not linked to a $3^{*}$-face then $\omega^{*}(v)=7-7=0$ by $(\mathrm{R} 2.2)$.

- If $k \geq 4$, then $\omega(v)=11 k-26$. Observe by (C1), (C2) and definitions of $n^{*}(v)$ and of linked vertices that:

$$
m_{3}(v) \leq\left\lfloor\frac{k}{2}\right\rfloor \quad \text { and } \quad k-2 m_{3}(v) \geq n_{2}(v)+n^{*}(v)
$$




$$
k \geq 2 m_{3}(v)+n_{2}(v)+n^{*}(v)
$$

It follows by (R1.1), (R1.2) and Equation (2) that:

$$
\begin{aligned}
\omega^{*}(v) & =11 k-26-9 m_{3}(v)-n^{*}(v)-2 n_{2}(v) \\
& \geq 11 k-26-9 m_{3}(v)-\frac{9}{2} n^{*}(v)-\frac{9}{2} n_{2}(v) \\
& \geq 11 k-26-\frac{9}{2} k \\
& \geq \frac{13}{2} k-26 \\
& \geq 0
\end{aligned}
$$

Suppose that $f$ is a $k$-face. Then, $k=3$ or $k \geq 13$ by hypothesis.

- If $k \geq 13$, then $\omega^{*}(f)=\omega(f)=2 k-26 \geq 0$.

- If $k=3$, then $\omega(f)=-20$. Suppose $f=[r s t]$. By (C2), $f$ is not incident to a 2-vertex ; hence, $d(r) \geq 3, d(s) \geq 3, d(t) \geq 3$. By (C4) $f$ is incident to at most one $3^{*}$-vertex. Now, observe that if one of the vertices $r, s, t$ is a $\geq 4$-vertex, then by (R1.2) (R2.1) (R2.2) (R2.3) $\omega^{*}(f) \geq-20+9+5+6=0$. So assume $d(r)=d(s)=d(t)=3$ and let $r_{0}, s_{0}$, $t_{0}$ be the other neighbors of $r, s, t$, respectively. Suppose that $f$ is a $3^{*}$-face and let $r$ be its unique $3^{*}$-vertex. By (C6) none of $s$ and $t$ are linked to a $3^{*}$-face. Moreover $s_{0}$ and $t_{0}$ give 1 to $f$ by (R1.2) and (R2.3). Hence $\omega^{*}(f)=-20+5+2 \cdot 7+2 \cdot 1=1$. Finally assume that $f$ is not a $3^{*}$ face. By (C5) at most one of $r, s, t$ is linked to a $3^{*}$-face. Hence $\omega^{*}(f) \geq-20+6+2 \cdot 7=0$, by (R1.2), (R2.2) and (R2.3).

We proved that, for all $x \in V(H) \cup F(H), \omega^{*}(x) \geq 0$. This completes the proof of Theorem 2 .

\section{References}

[1] O.V. Borodin, D.G. Fon-Der Flaass, A.V. Kostochka, A. Raspaud and E. Sopena, Acyclic list 7-coloring of planar graphs, J. Graph Theory, 40(2):83-90, 2002.

[2] O.V. Borodin, A.N. Glebov, A. Raspaud and M.R. Salavatipour, Planar graphs without cycles of length 4 to 7 are 3-colorable, J. Combin. Theory, Ser. B 93 303-311, 2005.

[3] O.V. Borodin, On acyclic colorings of planar graphs, Discrete Math., (25):211-236, 1979.

[4] O.V. Borodin, Structural properties of plane graphs without adjacent triangles and an application to 3-colorings, J. Graph Theory, 2(21):183-186, 1996.

[5] M. Chen, W. Wang, Acyclic 5-choosability of planar graphs without 4-cycles, Discrete Math. 308(24):6216-6225, 2008.

[6] P. Erdôs, A.L. Rubin and H. Taylor, Choosability in graphs, Congr. Numer., (26):125-157, 1979.

[7] T.R. Jensen and B. Toft, Graph coloring problems, Wiley Interscience, 1995.

[8] M. Montassier, Acyclic 4-choosability of planar graphs with girth at least 5, Trends in Mathematics: Graph Theory in Paris, 299-310, 2007.

[9] M. Montassier, P. Ochem and A. Raspaud, On the acyclic choosability of graphs, J. Graph Theory, 51(4):281-300, 2006. 
[10] M. Montassier, A. Raspaud and W. Wang, Acyclic 4-choosability of planar graphs without cycles of specific length, Algorithms and Combinatorics, 26:473-491, 2006.

[11] C. Thomassen, Every planar graph is 5-choosable, J. Combin. Theory Ser. B, 62:180-181, 1994.

[12] M. Voigt, List colourings of planar graphs, Discrete Math., (120):215-219, 1993. 